\title{
The plant metacaspase AtMC1 in pathogen-triggered programmed cell death and aging: functional linkage with autophagy
}

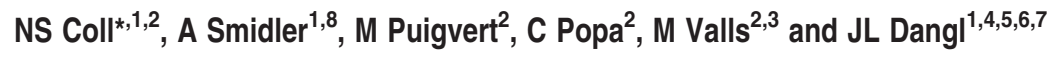

Autophagy is a major nutrient recycling mechanism in plants. However, its functional connection with programmed cell death (PCD) is a topic of active debate and remains not well understood. Our previous studies established the plant metacaspase AtMC1 as a positive regulator of pathogen-triggered PCD. Here, we explored the linkage between plant autophagy and AtMC1 function in the context of pathogen-triggered PCD and aging. We observed that autophagy acts as a positive regulator of pathogen-triggered PCD in a parallel pathway to AtMC1. In addition, we unveiled an additional, pro-survival homeostatic function of AtMC1 in aging plants that acts in parallel to a similar pro-survival function of autophagy. This novel pro-survival role of AtMC1 may be functionally related to its prodomain-mediated aggregate localization and potential clearance, in agreement with recent findings using the single budding yeast metacaspase YCA1. We propose a unifying model whereby autophagy and AtMC1 are part of parallel pathways, both positively regulating HR cell death in young plants, when these functions are not masked by the cumulative stresses of aging, and negatively regulating senescence in older plants.

Cell Death and Differentiation (2014) 21, 1399-1408; doi:10.1038/cdd.2014.50; published online 2 May 2014

An emerging theme in cell death research is that cellular processes thought to be regulated by linear signaling pathways are, in fact, complex. Autophagy, initially considered merely a nutrient recycling mechanism necessary for cellular homeostasis, was recently shown to regulate cell death, mechanistically interacting with components that control apoptosis. Deficient autophagy can result in apoptosis ${ }^{1-3}$ and autophagy hyper-activation can also lead to programmed cell death (PCD). ${ }^{4}$ In addition, the pro-survival function of autophagy is mediated by apoptosis inhibition and apoptosis mediates autophagy, although this cross-regulation is not fully understood. ${ }^{5}$

In plants, autophagy can also have both pro-survival and pro-death functions. Autophagy-deficient plants exhibit accelerated senescence, ${ }^{6-8}$ starvation-induced chlorosis, ${ }^{6,7,9}$ hypersensitivity to oxidative stress ${ }^{10}$ and endoplasmic reticulum stress. ${ }^{11}$ Further, autophagy-deficient plants cannot limit the spread of cell death after infection with tissue-destructive microbial infections. ${ }^{12,13}$ The plant phytohormone salicylic acid (SA) mediates most of these phenotypes. ${ }^{8}$ Autophagy has an essential, pro-survival role in situations where there is an increasing load of damaged proteins and organelles that need to be eliminated, that is, during aging or stress. Autophagy has an opposing, pro-death role during developmentally regulated cell death ${ }^{14,15}$ or during the pathogentriggered hypersensitive response PCD (hereafter, HR) that occurs locally at the site of attempted pathogen attack. ${ }^{16,17}$ The dual pro-death/pro-survival functions of plant autophagy remain a topic of active debate.

Also under scrutiny are possible novel functions of caspases and caspase-like proteins as central regulators of pro-survival processes. Caspases were originally defined as executioners of PCD in animals, but increasing evidence indicates that several caspases have non-apoptotic regulatory roles in cellular differentiation, motility and in the mammalian immune system. ${ }^{18-20}$

Yeast, protozoa and plants do not have canonical caspases, despite the occurrence of morphologically heterogeneous PCDs. ${ }^{21}$ More than a decade ago, distant caspase homologs termed metacaspases were identified in these organisms using structural homology searches. ${ }^{22}$ Metacaspases were classified into type I or type II metacaspases based on the presence or absence of an $\mathrm{N}$-terminal prodomain, reminiscent of the classification in animals into initiator/inflammatory or executioner caspases, respectively. Despite the architectural analogy between caspases and metacaspases, differences in their structure, function, activation and mode of action exist. ${ }^{23-25}$

Metacaspases mediate PCD in yeast, ${ }^{26-31}$ leishmania, ${ }^{32,33}$ trypanosoma ${ }^{34}$ and plants. ${ }^{24}$ We demonstrated that two type I metacaspases, AtMC1 and AtMC2, antagonistically regulate HR in Arabidopsis thaliana. ${ }^{35}$ Our work showed that AtMC1 is

\footnotetext{
${ }^{1}$ Department of Biology, University of North Carolina, Chapel Hill, NC 27599, USA; ${ }^{2}$ Centre for Research in Agricultural Genomics, Barcelona, Spain; ${ }^{3}$ Department of Genetics, Universitat de Barcelona, Barcelona, Spain; ${ }^{4}$ Howard Hughes Medical Institute, University of North Carolina, Chapel Hill, NC 27599 , USA; ${ }^{5}$ Curriculum in Genetics and Molecular Biology, University of North Carolina, Chapel Hill, NC 27599, USA; ${ }^{6}$ Department of Microbiology and Immunology, University of North Carolina, Chapel Hill, NC 27599, USA and ${ }^{7}$ Carolina Center for Genome Sciences University of North Carolina, Chapel Hill, NC, USA

*Corresponding author: NS Coll, Centre for Research in Agricultural Genomics, Campus UAB, Edifici CRAG, Bellaterra 08193, Barcelona, Spain. Tel: + 34 93 5636600; Fax: +34 93 5636601; E-mail: nuria.sanchez-coll@ cragenomica.es

${ }^{8}$ Current address: Department of Immunology and Infectious Diseases, Harvard School of Public Health, Boston, MA 02115, USA.

Abbreviations: PCD, programmed cell death; HR, hypersensitive response cell death; SA, salicylic acid; ROS, reactive oxygen species; FB1, fumonisin B1; NLR, nucleotide-binding domain and leucine-rich repeat containing; BTH, benzo(1,2,3)thiadiazole-7-carbothioic acid S-methyl ester

Received 06.2.14; revised 11.3.14; accepted 13.3.14; Edited by G Salvesen; published online 02.5.14
} 
a positive regulator of $\mathrm{HR}$ and that this function is mediated by its catalytic activity and negatively regulated by the AtMC1 $\mathrm{N}$-terminal prodomain. AtMC2 antagonizes AtMC1-mediated HR.

Besides AtMC2, new examples of metacaspases with a prolife/non-PCD role are emerging. Protozoan metacaspases are involved in cell cycle dynamics ${ }^{34,36-38}$ and cell proliferation. ${ }^{39}$ The yeast metacaspase Yca1 alters cell cycle dynamics ${ }^{40}$ and interestingly, is required for clearance of insoluble protein aggregates, thus contributing to yeast fitness. ${ }^{41}$

Here, we explore the linkage between plant autophagy and AtMC1 function in the context of pathogen-triggered HR and aging. Our data support a model wherein autophagy and AtMC1 are part of parallel pathways, both positively regulating $\mathrm{HR}$ cell death in young plants and negatively regulating senescence in older plants.

\section{Results}

Autophagy components and AtMC1 act additively to positively regulate HR. Autophagy is induced by activation of plant intracellular NLR (nucleotide-binding domain and leucine-rich repeat containing) immune receptors upon pathogen recognition, and thus can be a positive regulator of HR in Arabidopsis young leaves. ${ }^{16,17}$ To ascertain whether AtMC1- and autophagy-mediated HR are part of the same pathway, we crossed Arabidopsis atmc1 knockout plants ${ }^{35}$ to two different autophagy-deficient knockout mutants: atg $5^{42}$ and atg18a. $^{13}$ ATG5 and ATG18a are each required for autophagosome formation at different points of the autophagic pathway. ${ }^{7,43}$ We infected 2-week-old wild-type Col-0, atmc1, atg5, atg18a, atmc1 atg5 and atmc1 atg18a plants with Pseudomonas syringae pathovar tomato strain (Pto DC3000 expressing the type III effector avrRpm1 Pto DC3000(avrRpm1)). Recognition of AvrRpm1 triggers HR mediated by the intracellular NLR receptor RPM1. ${ }^{44} \mathrm{We}$ quantified HR using a single-cell death assay, ${ }^{35}$ and we observed suppression of RPM1-mediated HR both in atmc $1^{35}$ and in autophagy-deficient mutant plants. When combined, autophagy and atmc1 deficiencies had an additive effect on HR suppression (Figure 1a). Thus, autophagy and AtMC1 mediate independent pathways triggered by NLR activation that contribute to HR.

Using the same assay, we observed that the lack of AtMC2, a negative regulator of AtMC1-mediated HR cell death, ${ }^{35}$ has no effect on autophagy-mediated HR cell death (Supplementary Figure 1). In atmc1 and autophagy-deficient mutants, HR suppression does not result in increased susceptibility to Pto DC3000(avrRpm1), uncoupling HR and pathogen growth restriction. ${ }^{35}$ Thus, the additive HR suppression in atmc1 atg18a double mutants did not result in enhanced pathogen proliferation (Figure 1b).

We also investigated whether atmc1 mutants were defective in autophagy. Figure $1 \mathrm{c}$ and Supplementary Figure 2 show Col-0 and atmc1 transgenic plants expressing the autophagosome marker GFP-ATG8a with or without concanamycin A treatment. ${ }^{43}$ Plants lacking atg18a (or atg5) are defective in autophagosome formation. ${ }^{10,17,43}$ Atmc1 mutants displayed normal autophagosome formation (Figure 1c).

Recently, the plant cargo receptor NBR1 was demonstrated to be a selective autophagy marker that constitutively over-accumulates in autophagy-deficient plants. ${ }^{45}$ We performed immunoblot analysis of mock- or Pto DC3000 (avrRpm1)-treated plants using anti-NBR1 antisera to address whether selective autophagy was induced during $\mathrm{HR}$. We observed slightly increased NBR1 accumulation 12-h postinoculation in all lines tested (Figures 1d and e), indicating that selective autophagy is not induced after RPM1 activation at a time point when the HR cell death is complete (Figure 1). Atmc1 plants expressed wild-type NBR1 levels in either uninfected controls or following RPM1 activation, indicating that AtMC1 deficiency alone did not result in NBR1-mediated selective autophagy defects. As expected, atg18a and atmc1 atg18a mutants express higher NBR1 levels than wild-type plants because of defective selective autophagy. ${ }^{45}$ This NBR1 over-accumulation is more pronounced in atmc1 atg18 double mutants, indicating that AtMC1 may have a role in selective autophagy when bulk autophagy is defective.

SA accumulation negatively regulates the contribution of autophagy, but not of AtMC1, to RPM1-mediated HR. SID2 encodes the chloroplastic isochorismate synthase 1, the rate-limiting SA biosynthetic enzyme required for the increased accumulation of this phytohormone observed following pathogen recognition. ${ }^{46}$ To investigate if the HR suppression phenotypes observed in young autophagy- and atmc1-deficient plants were SA dependent, we quantified HR in wild-type, atmc1, atg18a, sid2, atmc1 atg18a, atmc1 sid2 and atg18a sid2 and atmc1 atg18a sid2 plants (Figure 2). Sid2 plants supported wild-type HR cell death levels, indicating that SA accumulation is dispensable for RPM1mediated HR. ${ }^{47}$ Interestingly, we observed that the loss of SA accumulation restores nearly wild-type levels of HR in atg18a, but not in atmc1 plants (Figure 2). This suggests that SA accumulation negatively regulates the contribution of autophagy to RPM1-mediated HR in atg18a sid2, but does not significantly regulate the AtMC1 contribution in atmc1 sid2. This observation also reinforces our hypothesis that autophagy and AtMC1 participate in separate HR signaling pathways. In atmc1 atg18a sid2 plants, the lack of SA accumulation reverts only partially $\mathrm{HR}$ suppression, indicating that the additive effects on HR observed in atmc1 atg18a cannot be solely explained by the sum of both deficiencies. It is worth noting that at the developmental stage used for the single-cell HR assay, atmc1, atg18a and atmc1 atg18a expressed essentially equivalent basal SA levels (Supplementary Figure 3).

The plant respiratory burst NADPH oxidase encoded by $A$ trbohD is required for the reactive oxygen species (ROS) burst downstream of RPM1 activation, but contributes only modestly to regulation of RPM1-mediated HR (Supplementary Figure 4). ${ }^{48}$ Consistent with these data, the lack of an NADPH-dependent ROS burst did not alter HR suppression in atmc1, atg18a or atmc1 atg18a mutants (Supplementary Figure 4), indicating that this ROS burst acts independently or upstream of AtMC1 and autophagy.

Autophagy components and AtMC1 act additively to negatively regulate senescence. Autophagy-deficient plants exhibit an early senescence phenotype, evidenced by premature leaf chlorosis. ${ }^{6-9}$ Interestingly, atmc1 mutants 
a

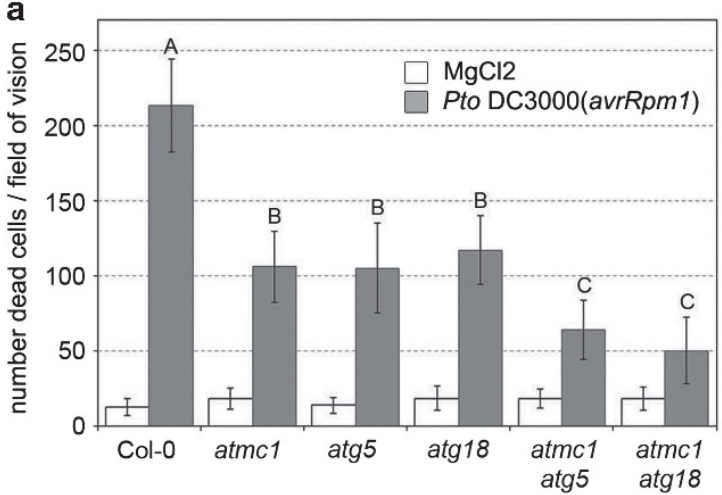

d

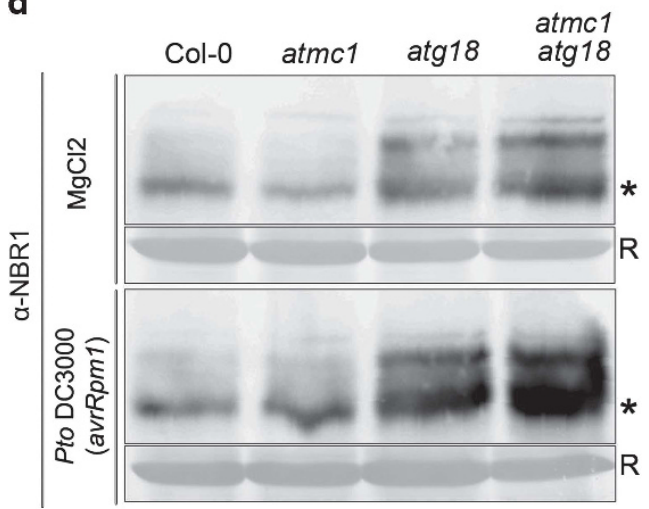

e

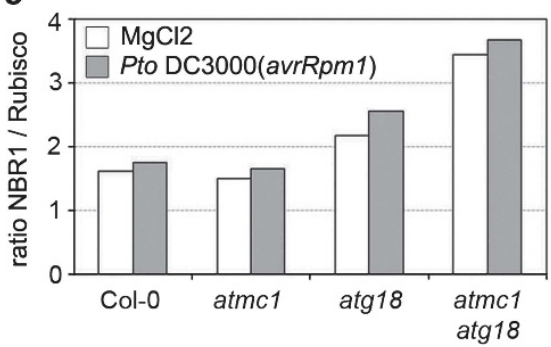

b

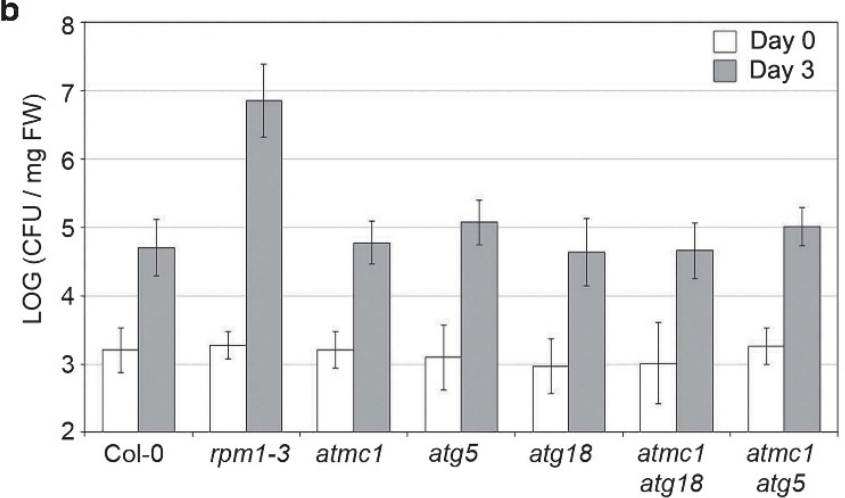

C

GFP-ATG8a

BF

merged

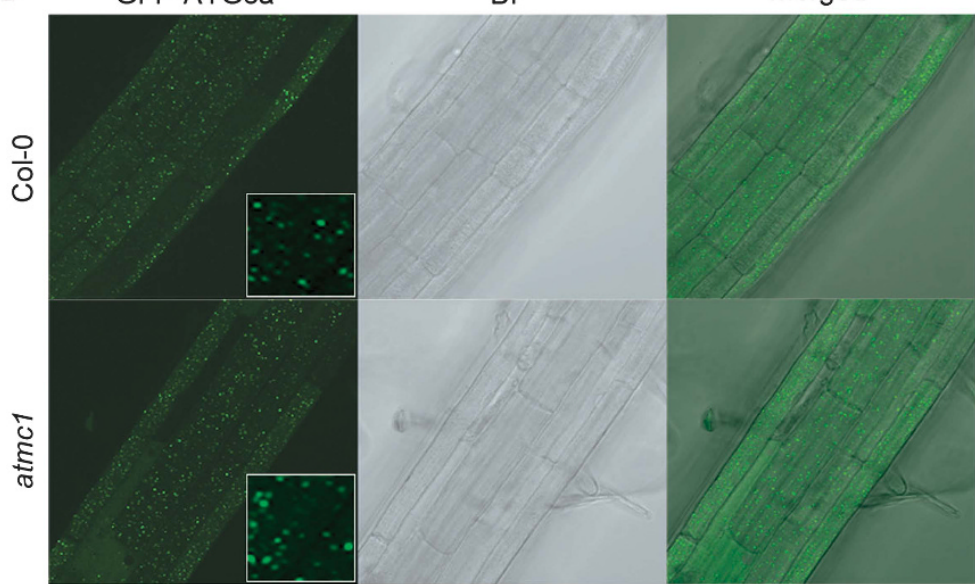

Figure 1 Autophagy components and AtMC1 act additively to positively regulate HR. (a) Two-week-old plants of the indicated phenotypes were vacuum infiltrated with 500000 colony-forming units (CFU)/ml of Pto DC3000(avrRpm1) or $\mathrm{MgCl}_{2}$. After $12 \mathrm{~h}$, plants were stained with the cell death dye Trypan blue. To quantify cell death, all dead cells per field of vision ( $\times 10$ magnification) were counted. Values correspond to the average of 20 leaves per genotype and treatment $\pm 2 \times \mathrm{S}$.E. Letters indicate a significant difference following post-ANOVA Student's $t$-test $(\alpha=0.05)$. The experiment is representative of three independent replicates. (b) Two-week-old plants of the indicated phenotypes were dip inoculated with $2.5 \times 10^{7} \mathrm{CFU} / \mathrm{ml}$ of Pto DC3000(avrRpm1). Bacterial growth was monitored at days 0 and 3 after infection. Values indicate the average of four samples per genotype $\pm 2 \times$ S.E. The experiment was repeated three times. (c) One-week-old transgenic Col-0 and atmc1 plants constitutively expressing GFP-ATG8 were treated with $1 \mu \mathrm{M}$ concanamycin A to allow autophagosome visualization in the vacuole of root cells using confocal microscopy. BF, bright field. Inlets show $\times 16$ magnifications of the central part of each root shown. (d) Western blot analysis of the NBR1 cargo receptor protein using plants of the noted genotypes treated as in (a). The band corresponding to NBR1 is marked with an asterisk. Coomassie-stained Rubisco (R) was used as a loading control. (e) Densitometry analysis of the samples in (d) using Multi Gauge (Fujfilm, ScienceLab 2005, version 3.0, Minato, Tokyo, Japan)

also senesce prematurely (Figure 3a). In atmc1 atg18a, this early senescence phenotype is enhanced and progresses faster than in either Col-0, atmc1 or atg18a plants (Supplementary Figure 5). These observations indicate that similar to autophagy, AtMC1 is also required for correctly timed leaf senescence and that autophagy and AtMC1 act additively on these processes.

Quantitative PCR analysis using the senescence marker $S A G 12^{49}$ confirmed the early senescence phenotype in 5-week-old atmc1, atg18a and atmc1 atg18a plants at the 
transcriptional level (Figure 3b). We did not detect any differences in SAG12 expression in 2-week-old plants. This indicates that the HR suppression phenotypes observed in atmc1, atg18a and atmc1 atg18a mutants cannot be explained by the early senescence onset, which occurs later.

Early senescence in autophagy-deficient plants, but not in atmc1 plants, requires SA accumulation. It was previously shown that the onset of early senescence and growth retardation in autophagy-deficient plants is correlated with SA hyper-accumulation. ${ }^{8}$ We confirmed and extended this result, showing that the lack of SA accumulation in sid2 atg18a largely reverts the early senescence phenotype of atg18 (Figure 3a). In contrast, AtMC1-regulated senescence processes occur independently of SA accumulation, as evidenced by the sid2 atmc1 early senescence phenotype. In addition, the fact that the lack of SA cannot fully revert the

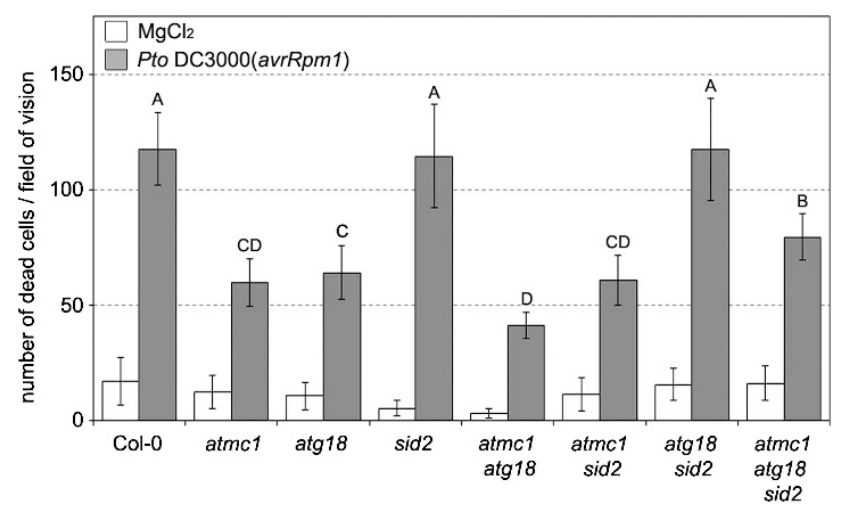

Figure 2 SA accumulation negatively regulates the autophagy contribution to RPM1-mediated HR, but does not significantly regulate the AtMC1 contribution. Two-week-old plants of the indicated phenotypes were vacuum infiltrated with 500000 colony-forming units (CFU)/ml of Pto DC3000(avrRpm1) or $\mathrm{MgCl}_{2}$. After $12 \mathrm{~h}$, plants were stained with the cell death dye Trypan blue. To quantify cell death, all dead cells per field of vision ( $\times 10$ magnification) were counted. Values indicate the average of 20 samples per genotype and treatment $\pm 2 \times$ S.E. Letters indicate a significant difference following post-ANOVA Student's $t$-test $(\alpha=0.05)$. The experiment is representative of three independent replicates extreme early senescence phenotype of atmc1 atg18a indicates that the additive effects on this phenotype cannot be solely explained by the sum of both deficiencies and that other - yet unknown - factors likely mediate this additivity.

atmc1 and atg18a mutants are hypersensitive to the SA agonist BTH and to externally generated ROS. We next treated atmc1, atg18a and atmc1 atg18a with either the SA agonist benzo(1,2,3)thiadiazole-7-carbothioic acid S-methyl ester (BTH) or different ROS-generating agents. BTH treatment resulted in leaf chlorosis in both atmc1 and atg18a, and this phenotype was enhanced in atmc1 atg18a but not in wildtype plants (Figure 4a). Leaf chlorosis was accompanied by increased ROS production and cell death (Figures 4b and c). The phenotype caused by BTH on these plants, grown under short-day conditions, is reminiscent of untreated plants grown 4 weeks under short-day conditions and then transferred to long-day conditions (Figure 3a). This suggests that lightdependent increases in SA accumulation trigger autophagy and AtMC1-mediated processes important for the proper remobilization of resources to reach a timely senescence.

To study the effect of ROS on autophagy or AtMC1regulated processes, plants were treated with rose bengal, methyl viologen or the fungal toxin fumonisin B1 (FB1) and cell death progression was visualized using Trypan blue (Figures $4 \mathrm{~d}$ and e). Methyl viologen treatment resulted in confined cell death in wild-type plants, modestly enhanced cell death in atmc1 and atg18a, and runaway cell death in atmc1 atg18a. These results suggest that both AtMC1 and autophagy have a function in downregulating the toxicity of ROS. Similar results were observed using rose bengal and FB1 as ROS accumulation triggers (Figure 4b). Together, these results indicate that the primary roles of autophagy and AtMC1 in older plants may be to protect the cells against the consequences of increasing ROS and SA levels during aging. Furthermore, aging autophagy- and atmc1-deficient plants cannot restrict cell death caused by the necrotrophic fungus Botrytis cinerea (Supplementary Figure 6). ${ }^{50}$ We infer from these results that autophagy and AtMC1 also act additively to limit cell death following necrotroph infection. a

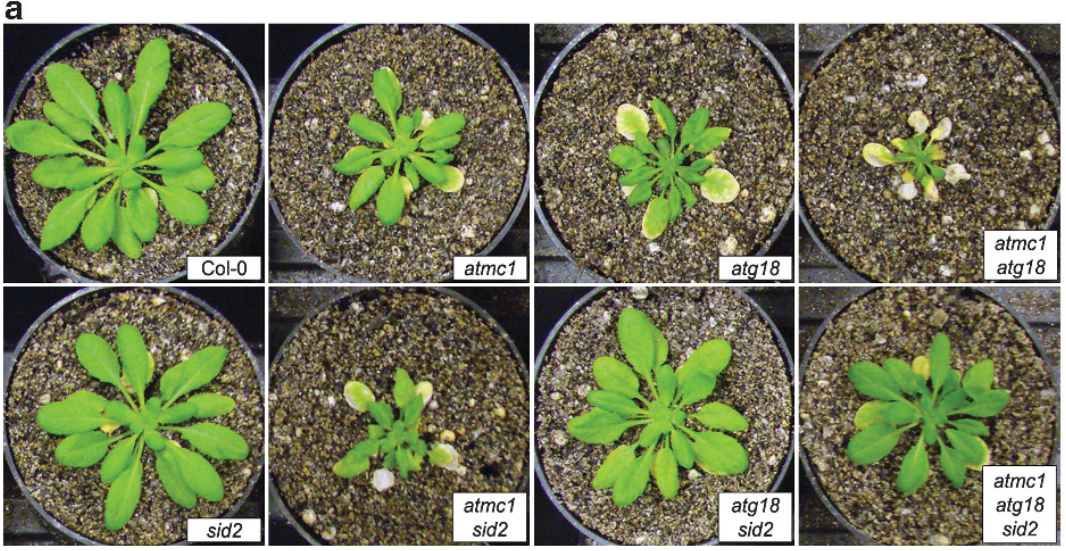

b

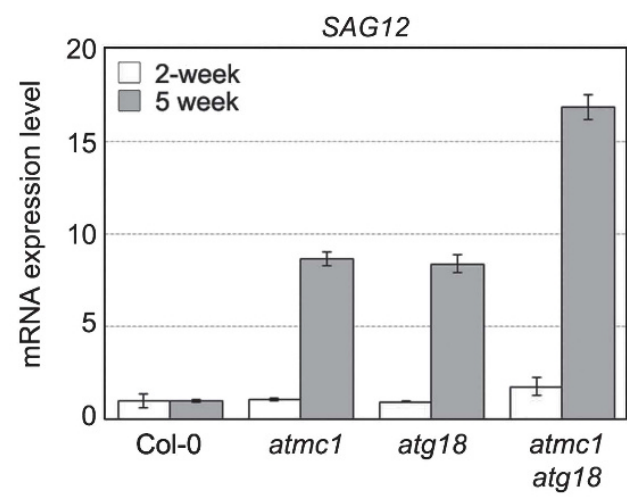

Figure 3 Autophagy components and AtMC1 act additively to negatively regulate senescence. (a) Early senescence was SA-dependent in autophagy-deficient plants but $\mathrm{SA}$-independent in atmc1 mutants. Pictures show plants grown for 3 weeks under short-day conditions and then transferred to long-day conditions for 4 additional weeks. (b) Quantitative real-time PCR analysis of the senescence marker gene SAG12 in 2- and 5-week-old plants of the indicated genotypes, normalized to EF-1 $\alpha$. The S.E. was calculated from three samples per genotype and the experiment was performed three times 
a

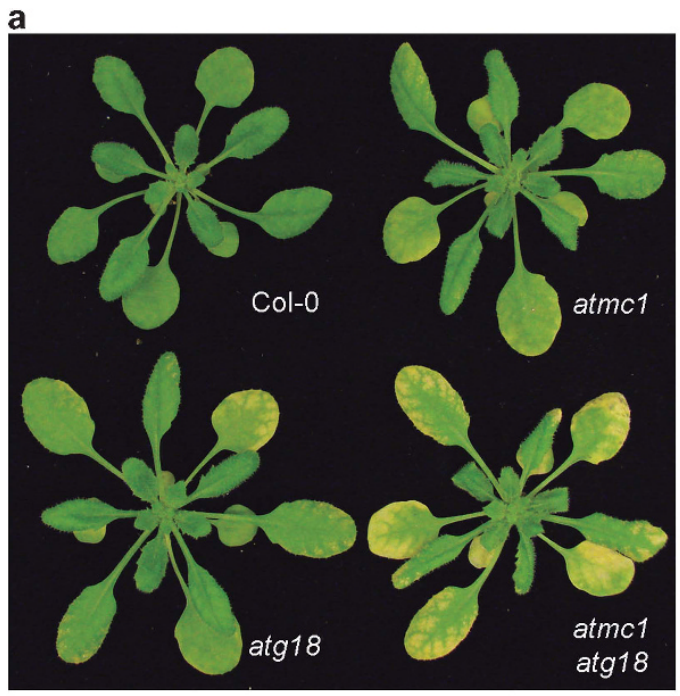

b

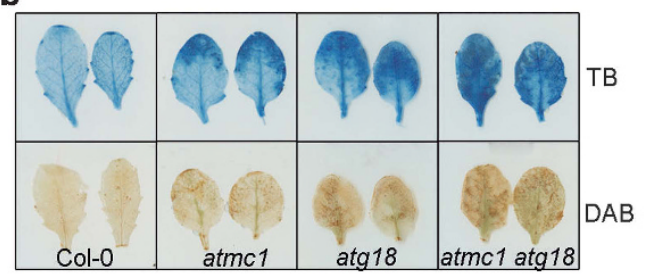

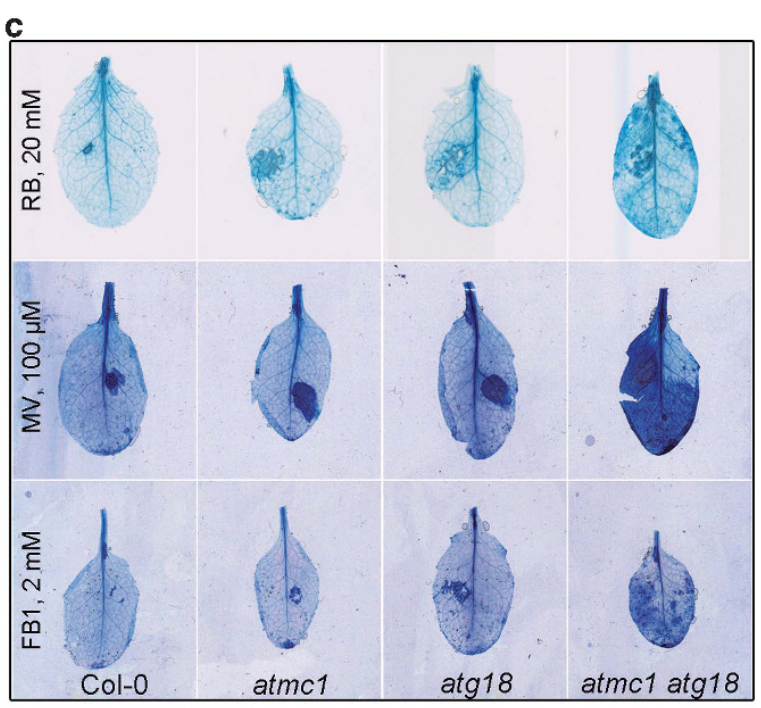

d

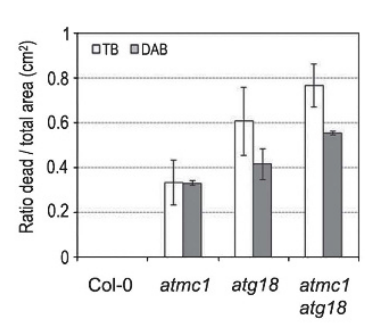

Figure 4 Atmc1 and atg18a mutants are hypersensitive to the SA agonist BTH and to externally generated ROS. (a) Pictures of representative 4-week-old plants grown under short-day conditions, 4 days after $300 \mu \mathrm{M}$ BTH treatment. (b) Representative leaves of plants treated as in (a) were stained with Trypan blue (TB, upper panel) or with 3,3-diamino-benzidine (DAB, lower panel) to visualize cell death and $\mathrm{H}_{2} \mathrm{O}_{2}$ accumulation, respectively. (c) Quantification of cell death and $\mathrm{H}_{2} \mathrm{O}_{2}$ accumulation in (b) by measuring the stained area (excluding the central vein) relative to the whole area of the leaf. (d) Pictures of representative 4-week-old plants $24 \mathrm{~h}$ after treatment with the ROS donors rose bengal (RB), methyl viologen (MV), the fungal toxin FB1, stained with Trypan blue to visualize cell death. (d and e) Quantification of cell death in (d) performed as in (c)

A fraction of full-length AtMC1 localizes to insoluble aggregates. The budding yeast Saccharomyces cerevisiae expresses a single type I metacaspase (Yca1), which mediates catalytic site-dependent PCD in this organism. ${ }^{26-31}$ However, Yca1 also can be localized to insoluble protein aggregates where it promotes aggregate clearance independent of the Yca1 catalytic site. ${ }^{41}$ Yca1 localization in protein aggregates is mediated by its $\mathrm{N}$-terminal putative prodomain. We hypothesized that AtMC1 may also target protein aggregates and mediate its clearance, independent of its pro-death role during $\mathrm{HR}$. Such a function could explain the early senescence and ROS/SA hypersensitivity of atmc1 plants. Furthermore, it could account for the observed enhancement of the SA and ROS sensitivity phenotypes of atmc1 atg18a, since those plants would lack two complementary pro-life processes required to cope with the strains of aging.

We studied AtMC1 subcellular localization in plants conditionally overexpressing AtMC1-HA (Figure 5a). ${ }^{35}$ Total protein extract $(\mathrm{T})$ contained equal amounts of full-length and cleaved, presumably active AtMC1 (Figure 5a, left). Most of the cleaved AtMC1 localized in the soluble fraction (S), whereas full-length AtMC1 was also present in the microsomal/insoluble fraction $(M+A)$. Subsequent solubilization of the microsomal/insoluble fraction revealed that AtMC1, in particular the full-length form, was insoluble $(A)$. This indicates that a fraction of full-length AtMC1 likely localizes to insoluble protein aggregates. We performed the same fractionation using plants expressing the catalytic dead version of AtMC1 (AtMC1-C99A-C220A-HA). ${ }^{35}$ The catalytic dead AtMC1 protein remained mostly insoluble. Taken together, these data indicate that at least part of the full-length AtMC1 localizes to insoluble aggregates independently of its catalytic activity, similar to yeast Yca1.

We also tested AtMC1 localization when expressed under the control of its native promoter (atmc1 pAtMC1::AtMC1-HA) using untreated or pathogen-treated young plants and older plants. Figure $5 \mathrm{~b}$ shows that natively expressed AtMC1 protein accumulation is induced by pathogen-triggered HR cell death and aging. As expected, AtMC1 aggregate localization reaches its maximum in aging plants.

Subsequently, we analyzed aggregate content in Col-0, atmc1, atg18a and atmc1 atg18a under basal (Figure 5d), pathogen-induced cell death and aging conditions using the total and soluble fractions as a loading control (Figure 5c). Early senescing atmc1 and atg18a mutants showed a higher aggregate content than wild-type plants. In atmc1 atg18a plants, aggregate over-accumulation was even more marked as expected from their additive phenotypes (Figure $5 d$ ). We hypothesize that localization mediates clearance of insoluble 


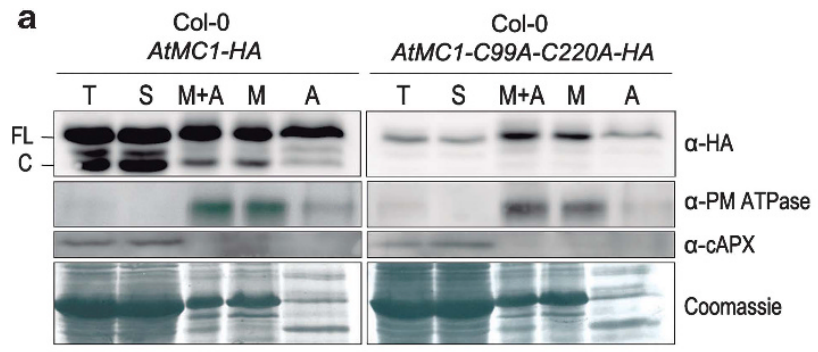

b

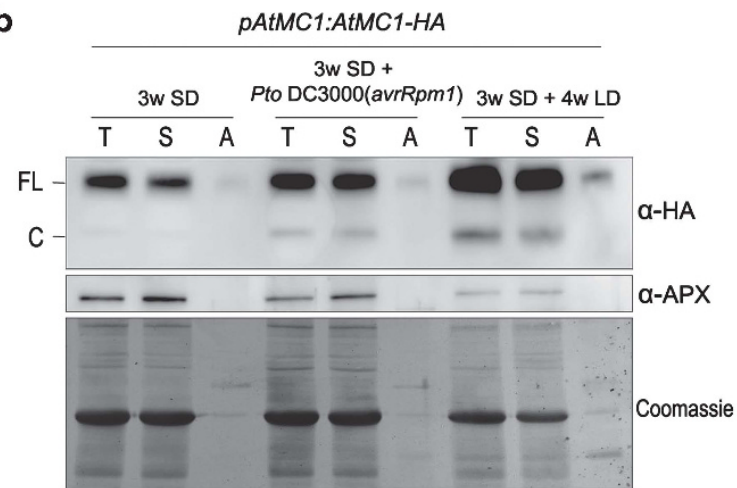

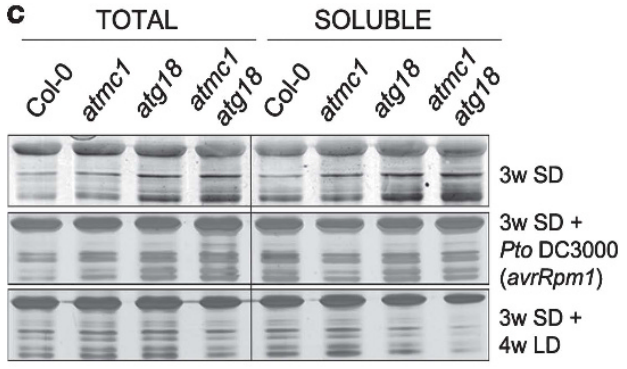

d

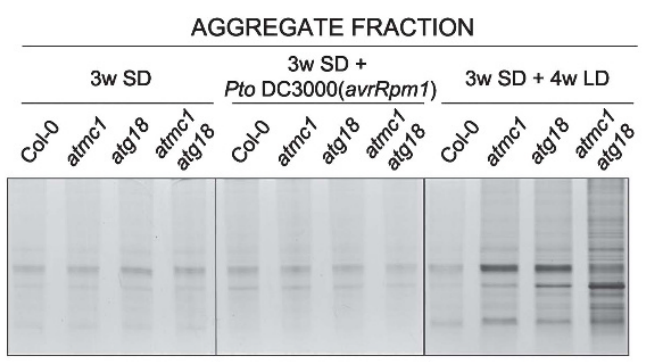

Figure 5 A fraction of full-length AtMC1 localizes to insoluble aggregates independent of its catalytic activity, contributing to aggregate clearance. (a) Protein extracts of 4-week-old Col-0 plants conditionally overexpressing AtMC1-HA (left) and AtMC1-C99AC220A-HA (right) were subjected to cellular fractionation. Total protein extract (T) was fractionated into a supernatant containing the soluble proteins $(S)$ and a pellet, containing microsomal proteins and aggregates $(S+A)$. This pellet was further fractionated into a supernatant, containing most of the microsomal proteins $(\mathrm{M})$, and a pellet, containing insoluble protein aggregates (A). After separation on an SDS-PAGE gel, the fractions were either Coomassie-stained or analyzed by immunoblot using anti-HA, anti-cytosolic ascorbate peroxidase (cAPX) and anti-plasma membrane (PM) $\mathrm{H}^{+}$ATPase. The HA antibody recognized full-length AtMC1 (FL) and cleaved, putatively active AtMC1 (C). (b) atmc1 pAtMC1::AtMC1-HA plants were grown for 3 weeks under short-day conditions (3w SD), treated with $500000 \mathrm{CFU} / \mathrm{ml}$ of Pto DC3000(avrRpm1) (3w SD + Pto DC3000(avrRpm1)) or transferred to long-day conditions (3w SD + 4w LD) and western blot analysis using anti-HA antibody or anti-cAPX was performed after fractionation into total (T), soluble (S) and insoluble aggregate (A) fractions. (c and d) Silver stains of total, soluble (c) and insoluble aggregate fractions (d) of plants of the indicated genotypes treated as in (b)

aggregates and thus contributes to cellular homeostasis and stress responses in a process that acts genetically in parallel to autophagy. This function is independent of, and does not preclude, the pro-death catalytic activity-dependent function of AtMC1 during HR cell death, which is most evident in young, non-stressed tissues.

\section{Discussion}

Autophagy and AtMC1 act in separate pathways as positive regulators of pathogen-triggered HR cell death. We previously demonstrated that AtMC1 is a positive regulator of $\mathrm{HR}$ cell death triggered by activation of different plant intracellular NLR innate immune receptors. ${ }^{35} \mathrm{~A}$ similar pro-death function was reported for autophagy. ${ }^{16,17}$ These findings were in sharp contrast to other studies, where autophagy was proposed as a pro-survival mechanism during HR cell death in plants. ${ }^{8,51,52}$ These apparent discrepancies can be reconciled in a model where autophagy has a pro-death role locally in the HR site, whereas in the surrounding uninfected tissue, autophagy promotes survival, protecting cells beyond the HR site from unnecessary damage. ${ }^{53,54}$ Signaling gradients that establish cell death control borders at sites of pathogen recognition have been demonstrated in plants. ${ }^{48,55-58}$ Importantly, the studies that reported a pro-survival role of autophagy during pathogentriggered HR cell death used relatively old plants. ${ }^{8,51,52}$
With age, autophagy mutants become prematurely senescent and accumulate high levels of ROS that can drive accumulation of SA, potentially increasing their vulnerability to ER stress. Activation of defense responses upon infection may further destabilize the already altered homeostasis in autophagy mutants, rendering them unable to restrict cell death. Consistent with this proposal, prevention of SA accumulation suppresses premature senescence and runaway cell death after pathogen infection in $\operatorname{atg} 5 .^{8}$

We therefore assayed young autophagy mutant plants treated with low-dose bacterial inocula more closely mimicking natural infections to avoid the unwanted effects of combinatorial stresses. Our data confirm previous findings defining autophagy as a positive regulator of $\mathrm{HR}^{16,17}$ Autophagy and AtMC1 act separately to contribute to HR, as evidenced by the further suppression of cell death in atmc1 atg18a. However, the independent pathways thus defined cannot account for full HR, as cell death suppression in the double mutant is incomplete. Hence, there must exist (an)other pathway(s), which account for the remaining HR.

The idea that AtMC1 and autophagy function in separate pathways during HR is supported by the fact that they are differentially regulated. The metacaspase AtMC2 negatively regulates AtMC $1^{35}$ but not autophagy. SA mediates the prodeath function of autophagy, but not of AtMC1. In fact, SA is a negative regulator of the combined contributions to HR regulated by AtMC1 and undefined contributors to $\mathrm{HR}$, as 
illustrated by the nearly complete recovery of HR in atg18a sid2 and the partial recovery of HR in atmc1 atg18a sid2. The recovery of $\mathrm{HR}$ in atg18a sid2 is not due to altered basal SA levels in these mutants. Our data are in agreement with previous findings establishing that SA can act as a negative regulator of $\mathrm{HR} .{ }^{59}$ Furthermore, our results are consistent with the idea that autophagy can be both a positive and a negative regulator of $\mathrm{HR}$ depending on the spatio-temporal context (HR site versus adjacent tissues or young versus old tissue). ${ }^{17,54}$ Finally, our data also show that HR suppression phenotypes in atmc1, atg18a and atmc1 atg18a is not accompanied by altered bacterial growth in any of these lines, further decoupling HR from pathogen growth restriction. ${ }^{35}$

The suppressed cell death phenotype in plants lacking AtMC1 is not due to defective autophagy. In order to explore the role of selective autophagy in pathogen-triggered HR and the possible linkage of AtMC1 to this process, we used the recently identified NBR1 autophagosome cargo protein marker. ${ }^{45}$ Autophagy-deficient mutants accumulate higher NBR1 basal levels than wild-type, ${ }^{45}$ which are further increased during the HR onset after RPM1 activation. This indicates that NBR1-mediated degradation of target proteins by autophagy may have an important role in HR cell death, perhaps contributing to vacuolar collapse.

Autophagy and AtMC1 independently control timely senescence in aging plants. Considering that autophagy has a main role in nutrient recycling, ${ }^{6,7,9}$ it is not surprising that autophagy-deficient plants are prematurely senescent. ${ }^{6-8}$ Furthermore, SA levels increase during senescence; this increase has been proposed to accelerate senescence once initiated. ${ }^{60}$ Autophagy mutants start accumulating SA at an earlier developmental stage than the wild-type $e^{8,13}$ and this over-accumulation underlies their premature senescent phenotype, as SA removal in these mutants results in normal timing of senescence. ${ }^{8}$

Besides its role in senescence, SA, in conjunction with ROS, is a potent defense regulator during infection. ${ }^{61,62}$ Treatment with the SA analog BTH causes chlorosis, ROS hyperaccumulation and cell death in autophagy-deficient plants, but not in wild-type plants. This hypersensitivity could result from accumulation of damaged proteins and organelles in these plants because of impaired autophagy-dependent recycling, which renders them less able to cope with further stress. Like autophagy-deficient plants, atmc1 plants are prematurely senescent and hypersensitive to BTH, ROS and necrotrophic fungi. In atmc1 atg18a plants, this phenotype is enhanced, indicating that the proteins act independently to downregulate these responses. Thus, AtMC1 has an additional, pro-survival homeostatic function in aging plants that acts in parallel to a similar pro-survival function of autophagy in aging.

A possible role of AtMC1 in protein aggregate clearance. Our data show that a fraction of the total full-length AtMC1 localizes to insoluble protein aggregates and this accumulation increases with age. Similar to yeast, aggregate localization of AtMC1 is also mediated by its $\mathrm{N}$-terminal prodomain, and AtMC1 localization to protein aggregates does not require its catalytic activity. Furthermore, atmc1 and atg18a plants, and to a further extent atmc1 atg18a, over-accumulate insoluble protein aggregates with age, which may be the cause of their premature senescence. The observed additive effects corroborate our notion that both pathways act independently to restrict insoluble protein aggregate accumulation.

Our hypothesis that AtMC1 functions in aggregate clearance is supported by the autophagy-like phenotypes of aging atmc1 null mutants: premature senescence and ROS hypersensitivity AtMC1-mediated aggregate clearance and autophagy could constitute two complementary processes controlling cellular homeostasis during stress responses and aging by virtue of their ability to eliminate accumulated cellular debris.

\section{A proposed model integrating the dual pro-death/ pro-survival functions of AtMC1 and autophagy at different developmental stages. In young plants, we defined pro- death functions for autophagy and AtMC1 in HR control, as these functions were not masked by the cumulative stresses of aging. Figure 6 a schematically shows a young plant cell undergoing HR after pathogen recognition. Under basal conditions, AtMC1 activation is prevented by the action of several negative regulators (AtMC2, LSD1 ${ }^{35}$ and probably other, unknown). Pathogen recognition leads to activation of intracellular NLR innate immune receptors, which results in local HR. In these circumstances, AtMC1 contributes to HR. Alternatively, enhanced auto-processing or processing by other metacaspases may contribute to accumulation of active AtMC1 in the cell. We speculate that the pro-death function of autophagy could be mediated by an active overload of the vacuole because of autophagy induction during HR, ultimately leading to vacuolar lysis. Interestingly, it has been recently reported that in Norway spruce the programmed vacuolar cell death that normally occurs in the}

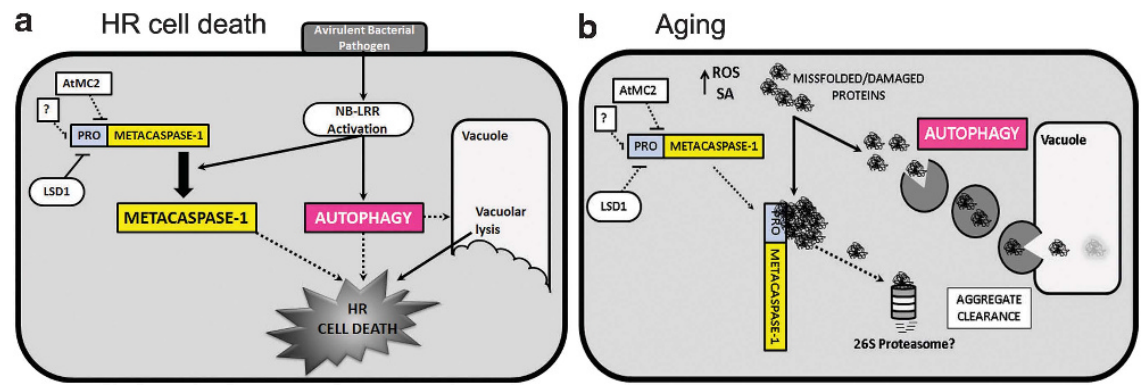

Figure 6 Proposed model integrating the dual pro-death/pro-survival functions of AtMC1 and autophagy at different developmental stages. (a) Pro-death functions of autophagy and AtMC1 in HR control in young plants. (b) Pro-survival role of autophagy and AtMC1 in aging cells 
embryo suspensor requires autophagy, which lies downstream of a type II metacaspase ${ }^{15}$ indicating that the interactions between the various cell death regulators may vary depending on the cellular scenario.

In aging cells, the pro-survival functions of AtMC1 and autophagy are revealed by the constant increase of damaged proteins and organelles that accumulate in the cell and require clearance (Figure 6b). In this developmental scenario, autophagy is induced to clear aggregates via their autophagosome-mediated delivery to the vacuole. We hypothesize that AtMC1 also contributes to this process by independently targeting aggregates and facilitating their degradation. Our genetic framework sets the stage for the elucidation of these mechanisms.

\section{Materials and Methods}

Plant materials and growth. All experiments were performed using Arabidopsis thaliana accession Col-0. Single mutant lines have been previously described elsewhere: atmc1 and atmc2, ${ }^{35}$ atg5 (SALK_020601), ${ }^{42}$ atg18a (GABI651D08), ${ }^{13}$ atrbohD, ${ }^{59}$ rpm1-3 ${ }^{63}$ and sid2/eds $16 .{ }^{46}$ Transgenic Col-0 35S:::GFP-ATG8a plants are described in Thompson et al. ${ }^{43}$ and atmc1 35S::GFP-ATG8a plants were obtained by transformation using the floral dip method. $^{64}$

Plants were grown under short-day conditions (9-h light, $21^{\circ} \mathrm{C}$; 15 -h dark, $18^{\circ} \mathrm{C}$ ) for most experiments. To study senescence, plants were transferred to long-day conditions (15-h light, $21^{\circ} \mathrm{C}$; 8-h dark, $18^{\circ} \mathrm{C}$ ) 3 or 4 weeks after germination.

Cell death assay and bacterial growth. Single HR cell death events after infection with Pto DC3000(avrRpm1) were quantified according to Coll et al..$^{35}$

Growth of Pto DC3000(avrRpm1) was tested using dip inoculations as previously described. ${ }^{65}$

Chemical treatments. Plants were grown 4 weeks under short-day conditions before treatment. For BTH treatment, plants were sprayed $300 \mu \mathrm{M}$ BTH supplemented with $0.005 \%$ Silwett.

To monitor oxidative stress, a $2 \mu \mathrm{l}$ drop of $100 \mu \mathrm{M}$ Methyl viologen, a $10 \mu \mathrm{l}$ drop of $2 \mathrm{mM}$ rose bengal or a $5 \mu \mathrm{l}$ drop of the necrotrophic fungal toxin FB1 were applied onto the abaxial surface of the leaf.

Stains. In order to visualize dead cells after chemical treatments, leaves were stained with Trypan blue as described. ${ }^{66,67} \mathrm{H}_{2} \mathrm{O}_{2}$ accumulation in leaves treated with $\mathrm{BTH}$ was visualized using $3,3^{\prime}$-diaminobenzidine staining as previously described. ${ }^{59}$ To quantify cell death and $\mathrm{H}_{2} \mathrm{O}_{2}$ accumulation from the pictures, total leaf area and cell death or stained area was measured using ImageJ (Bethesda, $\mathrm{MD}, \mathrm{USA}$ ), and the ratio (area of cell death/ total leaf area) was calculated.

Infection with the necrotroph Botrytis cinerea. Five-week-old plants were sprayed with $1 \times 10^{6}$ spores $/ \mathrm{ml}$ of Botrytis cinerea. Symptoms were visually followed for 1 week.

Total SA measurement. Total SA (free SA + glucose-conjugated SA, SAG) was measured as previously described, ${ }^{68}$ using as starting material $100 \mathrm{mg}$ of leaves from 2-week-old plants grown under short-day conditions (untreated).

RT-qPCR. Plant RNA was obtained from 2-week-old plants grown under shortday conditions or 5-week-old plants grown for 3 weeks under short-day and then transferred to long-day conditions. RNA was extracted using TRIzol (Life Technologies, Carlsbad, CA, USA) according to the manufacturer's instructions. RNA was treated 30 min with Ambion TURBO DNase (Life Technologies) to eliminate DNA contamination. Two microgram RNA was reverse transcribed using the Ambion RETROscript kit random decamers (Life Technologies).

RT-qPCR was performed using the Life Technologies SYBR Green PCR Master Mix in a total volume of $25 \mu \mathrm{l}: 12.5 \mu \mathrm{l}$ SYBR Green PCR Master Mix, $1 \mu \mathrm{l} \mathrm{CDNA}, 1 \mu \mathrm{l}$ forward primer $(10 \mu \mathrm{M}), 1 \mu \mathrm{l}$ reverse primer $2(10 \mu \mathrm{M})$ and $9.5 \mu \mathrm{l} \mathrm{H}_{2} \mathrm{O}$. The reaction was run at $95^{\circ} \mathrm{C}$ for $5 \mathrm{~min}$, followed by 40 cycles at $95^{\circ} \mathrm{C}$ for $15 \mathrm{~s}, 55^{\circ} \mathrm{C}$ for $30 \mathrm{~s}$ and $72{ }^{\circ} \mathrm{C}$ for $30 \mathrm{~s}$. Relative expression of SAG12 was calculated using the $\Delta \Delta \mathrm{Ct}$ method. ${ }^{69}$ SAG12 (At5g45890) expression was first normalized to expression of the housekeeping gene elongation factor1 $\alpha$ (At5g60390).

Confocal laser scanning microscopy. Seeds from transgenic lines expressing 35S::GFP-ATG8a in the Col-0 wild-type or atmc1 mutant backgrounds were surface sterilized in a $50 \%$ bleach and $0.2 \%$ Triton X-100 solution for $10 \mathrm{~min}$. Sterile seeds were plated onto solid MS medium plates (Murashige-Skoog Vitamin and Salt Mixture (Life Technologies), $2.4 \mathrm{mM}$ MES (pH 5.7) and 0.9\% Phyto Agar (Duchefa Biochemie, Haarlem, The Netherlands)). After 3 days vernalization at $4{ }^{\circ} \mathrm{C}$ in the dark, seedlings were grown for 1 week under short-day conditions. Seedlings were subsequently transferred to MS liquid medium (Murashige-Skoog Vitamin and Salt Mixture (Life Technologies), 2.4 mM MES (pH 5.7)) with or without $1 \mu \mathrm{M}$ concanamycin $\mathrm{A}$ and incubated for $15 \mathrm{~h}$ in the dark.

Roots were imaged using a Zeiss LSM 710 confocal laser scanning microscope (Zeiss, Oberkochen, Germany). All images were collected using a 40x/1.2NA C-Apochromat water immersion objective. Imaging of cells expressing GFP was performed using $480 \mathrm{~nm}$ excitation Scan parameters including pinhole, gain and offset were identical for each experiment to ensure image accuracy. Images were analyzed using the ZEN 2009 software (Zeiss).

Protein analysis. For the analysis of NBR1 protein accumulation, 2-week-old plants were vacuum infiltrated with $\sim 250000$ colony-forming units $/ \mathrm{ml}$ of Pto DC3000(avrRpm1). Leaf samples were snap frozen in liquid nitrogen $12 \mathrm{~h}$ after infection and mechanically ground in $250 \mu$ of plant extraction buffer $(20 \mathrm{mM}$ Tris (pH 7.5), $150 \mathrm{mM} \mathrm{NaCl}, 1 \mathrm{mM}$ EDTA, $1 \%$ Triton X-100 and 0.1\% SDS, $5 \mathrm{mM}$ DTT and 1:100 dilution of Protease Inhibitor Cocktail (Sigma, St. Louis, MO, USA)). Protein extract was centrifuged $15 \mathrm{~min}$ at $10000 \times g$ at $4^{\circ} \mathrm{C}$. The supernatants were collected, boiled on SDS-loading buffer (120 mM Tris, pH 6.8, 50\% glycerol, $6 \%$ SDS, $3 \mathrm{mM} \mathrm{DTT}$ and $1 \%$ Bromophenol blue) and separated on $7.5 \%$ SDS-PAGE gels. Immunoblot analysis was performed using a 1:1000 dilution of anti-NBR1 polyclonal antibody.

Cell fractionation. Plants were grown 4 weeks under short-day conditions. In all, $200 \mathrm{mg}$ of leaf tissue was ground in $4 \mathrm{ml}$ sucrose buffer $(20 \mathrm{mM}$ Tris (pH 8$)$, $0.33 \mathrm{M}$ sucrose, $1 \mathrm{mM}$ EDTA (pH 8) and 1:100 dilution of Protease Inhibitor Cocktail (Sigma)) and filtered through Miracloth (Millipore, Billerica, MA, USA). Samples were centrifuged $5 \mathrm{~min}$ at $4^{\circ} \mathrm{C}$ at $2000 \times \mathrm{g}$ to remove large particles. The supernatants were subsequently centrifuged $10 \mathrm{~min}$ at $4^{\circ} \mathrm{C}$ at $6000 \times \mathrm{g}$. An aliquot of the supernatant was collected representing the total protein fraction $(\mathrm{T})$ and the rest was centrifuged at $100000 \times g$ at $4^{\circ} \mathrm{C}$ for $90 \mathrm{~min}$. The supernatant (S) of this centrifugation was the soluble fraction. To separate microsomal proteins from protein aggregates in the pellet $(M+A)$, sucrose buffer containing $0.3 \%$ Triton X-100 was added. The pellet was redissolved by pipetting and incubation at $4{ }^{\circ} \mathrm{C}$ for $1 \mathrm{~h}$. Triton X-100-treated M+A was then centrifuged $50000 \times g$ at $4^{\circ} \mathrm{C}$ for $90 \mathrm{~min}$. The supernatant $(\mathrm{M})$ of this centrifugation represented the microsomal fraction, whereas the pellet $(A)$ corresponded to insoluble protein aggregates. Protein extracts were boiled on SDS-loading buffer and separated on $12 \%$ SDSPAGE gels. Gels were either Coomassie-stained or subjected to immunoblot analyisis using a 1:5000 dilution of anti-HA monoclonal antibody (3F10, Roche, Basel, Switzerland), 1:10000 anti-cAPX (Agrisera, Vännäs, Sweden) and anti-plasma membrane $\mathrm{H}^{+}$ATPase (Agrisera).

Alternatively, we used a modified version of the protocol described in Lee et al. ${ }^{41}$ obtaining similar results. Essentially, $1 \mathrm{~g}$ of plant tissue was ground in liquid nitrogen and $2 \mathrm{ml}$ of buffer $B$ was added (Buffer B: $50 \mathrm{mM}$ Tris, pH 7.5, $1 \mathrm{mM}$ EDTA, $1 \%$ glycerol, $0.1 \%$ Nonidet P-40 and protease inhibitor cocktail (Roche)). Cell debris was eliminated by passing the protein extract through a Miracloth filter (Millipore) and two sequential spins of 2000 and $3000 \times g$ at $4{ }^{\circ} \mathrm{C}$. Equal amounts of supernatant were collected (total) and centrifuged at $100000 \times \mathrm{g}$ at $4^{\circ} \mathrm{C}$ for $90 \mathrm{~min}$. The supernatant of this centrifugation corresponded to the soluble (S) fraction. The pellet was washed three times by adding buffer B supplemented with $2 \%$ Nonidet P-40 and centrifugation at $15000 \times g$ for $30 \mathrm{~min}$. The resulting insoluble protein aggregate fractions were resuspended in an equal volume of buffer $B(10 \times$ concentrated relative to the total and soluble fractions) and sonicated using a Bioruptor (Diagenode, Seraing, Belgium). In all, $6 \times$ loading buffer was then added and after boiling the samples for $10 \mathrm{~min}$ they were loaded on SDS-PAGE gels.

Silver staining. For silver staining, $40 \mu \mathrm{l}$ of cell equivalents of the total, soluble and aggregate fractions $(10 \times$ concentrated) were loaded on $12 \%$ SDS-PAGE gels. Gels were fixed for $1 \mathrm{~h}$ in a 50\% methanol, 37\% formaldehyde and $12 \%$ acetic acid 
solution. After three washes with $50 \%$ ethanol, gels were pre-treated 1 min with a $0.02 \%$ sodium thiosulfate solution, washed three times with water and stained $20 \mathrm{~min}$ in the dark with a $0.2 \%$ silver nitrate, $0.03 \%$ formaldehyde solution. Gels were then washed three times with water and treated with a $6 \%$ sodium carbonate $0.02 \%$ formaldehyde, $0.0005 \%$ sodium thiosulfate solution until the bands became visible. Gels were then washed for $5 \mathrm{~s}$ with water and a stop solution $(50 \%$ methano and $12 \%$ acetic acid) was added for 10 min. Once the reaction was stopped, gels were transferred to water for short-term storage.

\section{Conflict of Interest}

The authors declare no conflict of interest.

Acknowledgements. We thank S Svenning (University of Tromsø, Norway) for the NBR1 antisera, T Nürnberger (University Tübingen, Tübingen, Germany) for the atg18a mutant seeds and S Dinesh-Kumar (University California-Davis, CA, USA) for the atg5 mutant. We kindly thank R Vierstra (University Wisconsin-Madison, WI, USA) for critical reading of the manuscript and for sharing the Col-0 35S::GFPATG8a seeds with us. We also thank JL Crespo (Instituto de Bioquímica Vegetal y Fotosintesis, Spain) for sharing the ATG8 antisera and for valuable advices. This research was supported by NIH grant RO1GM057171 JLD and PCDMC-321738 from EU-Marie Curie Actions and BP B 00030 from the Catalan Government and to NSC. JLD is an HHMl investigator and this work was funded in part by the Howard Hughes Medical Institute and the Gordon and Betty Moore Foundation (GBMF3030).

1. Hara T, Nakamura K, Matsui M, Yamamoto A, Nakahara Y, Suzuki-Migishima R et al. Suppression of basal autophagy in neural cells causes neurodegenerative disease in mice. Nature 2006; 441: 885-889.

2. Komatsu M, Waguri S, Chiba T, Murata S, Iwata J, Tanida I et al. Loss of autophagy in the central nervous system causes neurodegeneration in mice. Nature 2006; 441: 880-884.

3. Takacs-Vellai K, Vellai T, Puoti A, Passannante M, Wicky C, Streit A et al. Inactivation of the autophagy gene bec-1 triggers apoptotic cell death in C. elegans. Curr Biol 2005; 15 1513-1517.

4. Gonzalez-Polo RA, Boya P, Pauleau AL, Jalil A, Larochette N, Souquere S et al. The apoptosis/autophagy paradox: autophagic vacuolization before apoptotic death. J Cell Sci 2005; 118(Pt 14): 3091-3102.

5. Gordy C, He YW. The crosstalk between autophagy and apoptosis: where does this lead? Protein Cell 2012. 3. 17-27.

6. Doelling JH, Walker JM, Friedman EM, Thompson AR, Vierstra RD. The APG8/12activating enzyme APG7 is required for proper nutrient recycling and senescence in Arabidopsis thaliana. J Biol Chem 2002; 277: 33105-33114.

7. Xiong Y, Contento AL, Bassham DC. AtATG18a is required for the formation of autophagosomes during nutrient stress and senescence in Arabidopsis thaliana. Plant $J$ 2005; 42: 535-546.

8. Yoshimoto K, Jikumaru Y, Kamiya Y, Kusano M, Consonni C, Panstruga R et al. Autophagy negatively regulates cell death by controlling NPR1-dependent salicylic acid signaling during senescence and the innate immune response in Arabidopsis. Plant Cell 2009; 21: 2914-2927.

9. Hanaoka H, Noda T, Shirano Y, Kato T, Hayashi H, Shibata D et al. Leaf senescence and starvation-induced chlorosis are accelerated by the disruption of an Arabidopsis autophagy gene. Plant Physiol 2002; 129: 1181-1193.

10. Xiong $Y$, Contento AL, Nguyen $P Q$, Bassham DC. Degradation of oxidized proteins by autophagy during oxidative stress in Arabidopsis. Plant Physiol 2007; 143: 291-299.

11. Liu Y, Burgos JS, Deng Y, Srivastava R, Howell SH, Bassham DC. Degradation of the endoplasmic reticulum by autophagy during endoplasmic reticulum stress in Arabidopsis. Plant Cell 2012; 24: 4635-4651.

12. Lai Z, Wang F, Zheng Z, Fan B, Chen Z. A critical role of autophagy in plant resistance to necrotrophic fungal pathogens. Plant J 2011; 66: 953-968.

13. Lenz HD, Haller E, Melzer E, Kober K, Wurster K, Stahl M et al. Autophagy differentially controls plant basal immunity to biotrophic and necrotrophic pathogens. Plant $\mathrm{J} 2011 ; 66$ : 818-830.

14. Kwon SI, Cho HJ, Jung JH, Yoshimoto K, Shirasu K, Park OK. The Rab GTPase RabG3b functions in autophagy and contributes to tracheary element differentiation in Arabidopsis. Plant J 2010; 64: 151-164.

15. Minina EA, Filonova LH, Fukada K, Savenkov El, Gogvadze V, Clapham D et al. Autophagy and metacaspase determine the mode of cell death in plants. J Cell Biol 2013; 203: 917-927.

16. Hofius D, Schultz-Larsen T, Joensen J, Tsitsigiannis DI, Petersen NH, Mattsson O et al. Autophagic components contribute to hypersensitive cell death in Arabidopsis. Cell 2009; 137: 773-783.

17. Kwon SI, Cho HJ, Kim SR, Park OK. The Rab GTPase RabG3b positively regulates autophagy and immunity-associated hypersensitive cell death in Arabidopsis. Plant Physio 2013; 161: 1722-1736.
18. Feinstein-Rotkopf $Y$, Arama E. Can't live without them, can live with them: roles of caspases during vital cellular processes. Apoptosis 2009; 14: 980-995.

19. Portela M, Richardson HE. Death takes a holiday-non-apoptotic role for caspases in cell migration and invasion. EMBO Rep 2013; 14: 107-108.

20. Rodrigue-Gervais IG, Saleh M. Caspases and immunity in a deadly grip. Trends Immuno 2013; 34: 41-49.

21. van Doorn WG, Beers EP, Dangl JL, Franklin-Tong VE, Gallois P, Hara-Nishimura I et al. Morphological classification of plant cell deaths. Cell Death Differ 2011; 18: 1241-1246.

22. Uren AG, O'Rourke $K$, Aravind LA, Pisabarro MT, Seshagiri S, Koonin EV et al. Identification of paracaspases and metacaspases: two ancient families of caspase-like proteins, one of which plays a key role in MALT lymphoma. Mol Cell 2000; 6: 961-967.

23. McLuskey K, Rudolf J, Proto WR, Isaacs NW, Coombs GH, Moss CX et al. Crystal structure of a Trypanosoma brucei metacaspase. Proc Natl Acad Sci USA 2012; 109: 7469-7474.

24. Tsiatsiani L, Van Breusegem F, Gallois P, Zavialov A, Lam E, Bozhkov PV. Metacaspases. Cell Death Differ 2011; 18: 1279-1288.

25. Wong AH, Yan C, Shi Y. Crystal structure of the yeast metacaspase Yca1. J Biol Chem 2012; 287: 29251-29259.

26. Gonzalez IJ, Desponds C, Schaff C, Mottram JC, Fasel N. Leishmania major metacaspase can replace yeast metacaspase in programmed cell death and has arginine-specific cysteine peptidase activity. Int J Parasitol 2007; 37: 161-172.

27. Ivanovska I, Hardwick JM. Viruses activate a genetically conserved cell death pathway in a unicellular organism. J Cell Biol 2005; 170: 391-399.

28. Khan MA, Chock PB, Stadtman ER. Knockout of caspase-like gene, YCA1, abrogates apoptosis and elevates oxidized proteins in Saccharomyces cerevisiae. Proc Natl Acad Sci USA 2005; 102: 17326-17331.

29. Madeo F, Herker E, Maldener C, Wissing S, Lachelt S, Herlan M et al. A caspase-related protease regulates apoptosis in yeast. Mol Cell 2002; 9: 911-917.

30. Mazzoni C, Herker E, Palermo V, Jungwirth $\mathrm{H}$, Eisenberg T, Madeo $\mathrm{F}$ et al. Yeast caspase 1 links messenger RNA stability to apoptosis in yeast. EMBO Rep 2005; 6: 1076-1081.

31. Silva RD, Sotoca R, Johansson B, Ludovico P, Sansonetty F, Silva MT et al. Hyperosmotic stress induces metacaspase- and mitochondria-dependent apoptosis in Saccharomyces cerevisiae. Mol Microbiol 2005; 58: 824-834.

32. Lee N, Gannavaram S, Selvapandiyan A, Debrabant A. Characterization of metacaspases with trypsin-like activity and their putative role in programmed cell death in the protozoan parasite Leishmania. Eukaryot Cell 2007; 6: 1745-1757.

33. Zalila H, Gonzalez IJ, El-Fadili AK, Delgado MB, Desponds C, Schaff C et al. Processing of metacaspase into a cytoplasmic catalytic domain mediating cell death in Leishmania major. Mol Microbiol 2011; 79: 222-239.

34. Laverriere M, Cazzulo JJ, Alvarez VE. Antagonic activities of Trypanosoma cruzi metacaspases affect the balance between cell proliferation, death and differentiation. Cell Death Differ 2012; 19: 1358-1369.

35. Coll NS, Vercammen D, Smidler A, Clover C, Van Breusegem F, Dangl JL et al. Arabidopsis type I metacaspases control cell death. Science 2010; 330: 1393-1397.

36. Ambit A, Fasel N, Coombs GH, Mottram JC. An essential role for the Leishmania major metacaspase in cell cycle progression. Cell Death Differ 2008; 15: 113-122.

37. Helms MJ, Ambit A, Appleton P, Tetley L, Coombs GH, Mottram JC. Bloodstream form Trypanosoma brucei depend upon multiple metacaspases associated with RAB11-positive endosomes. J Cell Sci 2006; 119(Pt 6): 1105-1117.

38. Proto WR, Castanys-Munoz E, Black A, Tetley L, Moss CX, Juliano L et al. Trypanosoma brucei metacaspase 4 is a pseudopeptidase and a virulence factor. J Biol Chem 2011; 286: 39914-39925

39. Szallies A, Kubata BK, Duszenko M. A metacaspase of Trypanosoma brucei causes loss of respiration competence and clonal death in the yeast Saccharomyces cerevisiae. FEBS Lett 2002; 517: 144-150.

40. Lee RE, Puente LG, Kaern M, Megeney LA. A non-death role of the yeast metacaspase: Yca1p alters cell cycle dynamics. PLoS One 2008; 3: e2956.

41. Lee RE, Brunette S, Puente LG, Megeney LA. Metacaspase Yca1 is required for clearance of insoluble protein aggregates. Proc Natl Acad Sci USA 2010; 107: 13348-13353.

42. Wang Y, Nishimura MT, Zhao T, Tang D. ATG2, an autophagy-related protein, negatively affects powdery mildew resistance and mildew-induced cell death in Arabidopsis. Plant $J$ 2011; 68: 74-87.

43. Thompson AR, Doelling JH, Suttangkakul A, Vierstra RD. Autophagic nutrient recycling in Arabidopsis directed by the ATG8 and ATG12 conjugation pathways. Plant Physiol 2005; 138: $2097-2110$

44. Debener T, Lehnackers H, Arnold M, Dangl JL. Identification and molecular mapping of a single Arabidopsis thaliana locus determining resistance to a phytopathogenic Pseudomonas syringae isolate. Plant J 1991; 1: 289-302.

45. Svenning S, Lamark T, Krause K, Johansen T. Plant NBR1 is a selective autophagy substrate and a functional hybrid of the mammalian autophagic adapters NBR1 and p62/ SQSTM1. Autophagy 2011; 7: 993-1010.

46. Wildermuth MC, Dewdney J, Wu G, Ausubel FM. Isochorismate synthase is required to synthesize salicylic acid for plant defence. Nature 2001; 414: 562-565

47. Tsuda K, Sato M, Glazebrook J, Cohen JD, Katagiri F. Interplay between MAMP-triggered and SA-mediated defense responses. Plant J 2008; 53: 763-775.

48. Torres MA, Jones JD, Dangl JL. Pathogen-induced, NADPH oxidase-derived reactive oxygen intermediates suppress spread of cell death in Arabidopsis thaliana. Nat Genet 2005; 37: 1130-1134. 
49. Noh YS, Amasino RM. Identification of a promoter region responsible for the senescencespecific expression of SAG12. Plant Mol Biol 1999; 41: 181-194.

50. Govrin EM, Levine A. The hypersensitive response facilitates plant infection by the necrotrophic pathogen Botrytis cinerea. Curr Biol 2000; 10: 751-757.

51. Liu Y, Schiff M, Czymmek K, Talloczy Z, Levine B, Dinesh-Kumar SP. Autophagy regulates programmed cell death during the plant innate immune response. Cell 2005; 121: 567-577.

52. Patel S, Dinesh-Kumar SP. Arabidopsis ATG6 is required to limit the pathogen-associated cell death response. Autophagy 2008; 4: 20-27.

53. Hayward AP, Dinesh-Kumar SP. What can plant autophagy do for an innate immune response? Annu Rev Phytopathol 2011; 49: 557-576.

54. Hofius D, Munch D, Bressendorff S, Mundy J, Petersen M. Role of autophagy in disease resistance and hypersensitive response-associated cell death. Cell Death Differ 2011; 18: 1257-1262.

55. Costet L, Cordelier S, Dorey S, Baillieul F, Fritig B, Kauffmann S. Relationship between localized acquired resistance (LAR) and the hypersensitive response (HR): HR is necessary for LAR to occur and salicylic acid is not sufficient to trigger LAR. Mol Plant Microbe Interact 1999; 12: 655-662.

56. Dorey S, Baillieul F, Pierrel MA, Saindrenan P, Fritig B, Kauffmann S. Spatial and temporal induction of cell death, defense genes, and accumulation of salicylic acid in tobacco leaves reacting hypersensitively to a fungal glycoprotein elicitor. Mol Plant Microbe Interact 1997; 10: $646-655$.

57. Roberts M, Tang S, Stallmann A, Dangl JL, Bonardi V. Genetic requirements for signaling from an autoactive plant NB-LRR intracellular innate immune receptor. PLoS Genet 2013; 9: e1003465.

58. Shirasu K, Nakajima H, Rajasekhar VK, Dixon RA, Lamb C. Salicylic acid potentiates an agonist-dependent gain control that amplifies pathogen signals in the activation of defense mechanisms. Plant Cell 1997; 9: 261-270.
59. Torres MA, Dangl JL, Jones JD. Arabidopsis gp91phox homologues AtrbohD and AtrbohF are required for accumulation of reactive oxygen intermediates in the plant defense response. Proc Natl Acad Sci USA 2002; 99: 517-522.

60. Abreu ME, Munne-Bosch S. Photo- and antioxidant protection and salicylic acid accumulation during post-anthesis leaf senescence in Salvia lanigera grown under Mediterranean climate. Physiol Plant 2007; 131: 590-598.

61. Lawton KA, Friedrich L, Hunt M, Weymann K, Delaney $\mathrm{T}$, Kessmann $\mathrm{H}$ et al. Benzothiadiazole induces disease resistance in Arabidopsis by activation of the systemic acquired resistance signal transduction pathway. Plant J 1996; 10: 71-82.

62. Yang Y, Shah J, Klessig DF. Signal perception and transduction in plant defense responses. Genes Dev 1997; 11: 1621-1639.

63. Grant MR, Godiard L, Straube E, Ashfield T, Lewald J, Sattler A et al. Structure of the Arabidopsis RPM1 gene enabling dual specificity disease resistance. Science 1995; 269: 843-846.

64. Clough SJ, Bent AF. Floral dip: a simplified method for Agrobacterium-mediated transformation of Arabidopsis thaliana. Plant J 1998; 16: 735-743.

65. Tornero P, Dangl JL. A high-throughput method for quantifying growth of phytopathogenic bacteria in Arabidopsis thaliana. Plant J 2001; 28: 475-481.

66. Keogh RC, Deverall BJ, McLeod S. Comparison of histological and physiological responses to Phakopsora pachyrhizi in resistant and susceptible soybean. Trans Br Mycol Soc 1980; 74: 329-333.

67. Koch E, Slusarenko A. Arabidopsis is susceptible to infection by a downy mildew fungus. Plant Cell 1990; 2: 437-445.

68. Bonardi V, Tang S, Stallmann A, Roberts M, Cherkis K, Dangl JL. Expanded functions for a family of plant intracellular immune receptors beyond specific recognition of pathogen effectors. Proc Natl Acad Sci USA 2011; 108: 16463-16468.

69. Livak KJ, Schmittgen TD. Analysis of relative gene expression data using real-time quantitative PCR and the 2(-delta delta C(T)) method. Methods 2001; 25: 402-408.

\section{Supplementary Information accompanies this paper on Cell Death and Differentiation website (http://www.nature.com/cdd)}

